\title{
Clinical Phase I/II trial to Investigate Preoperative Dose-Escalated Intensity-Modulated Radiation Therapy (IMRT) and Intraoperative Radiation Therapy (IORT) in patients with retroperitoneal soft tissue sarcoma: interim analysis
}

Falk Roeder ${ }^{1,2,8^{*}}$, Alexis Ulrich ${ }^{3}$, Gregor Habl ${ }^{2}$, Matthias Uhl², Ladan Saleh-Ebrahimi ${ }^{1,8}$, Peter E Huber ${ }^{1,2}$, Daniela Schulz-Ertner ${ }^{4}$, Anna V Nikoghosyan ${ }^{5}$, Ingo Alldinger ${ }^{3}$, Robert Krempien ${ }^{5}$, Gunhild Mechtersheimer ${ }^{6}$, Frank W Hensley ${ }^{2}$, Juergen Debus ${ }^{1,2}$ and Marc Bischof ${ }^{7}$

\begin{abstract}
Background: To report an unplanned interim analysis of a prospective, one-armed, single center phase I/II trial (NCT01566123).

Methods: Between 2007 and 2013, 27 patients (pts) with primary/recurrent retroperitoneal sarcomas (size $>5 \mathrm{~cm}$, $\mathrm{MO}$, at least marginally resectable) were enrolled. The protocol attempted neoadjuvant IMRT using an integrated boost with doses of 45-50 Gy to PTV and 50-56 Gy to GTV in 25 fractions, followed by surgery and IOERT (10-12 Gy). Primary endpoint was 5-year-LC, secondary endpoints included PFS, OS, resectability, and acute/late toxicity. The majority of patients showed high grade lesions (FNCLCC G1:18\%, G2:52\%, G3:30\%), predominantly liposarcomas (70\%). Median tumor size was $15 \mathrm{~cm}(6-31)$.

Results: Median follow-up was 33 months (5-75). Neoadjuvant IMRT was performed as planned (median dose $50 \mathrm{~Gy}, 26-55)$ in all except 2 pts (93\%). Gross total resection was feasible in all except one patient. Final margin status was R0 in $6(22 \%)$ and R1 in 20 pts (74\%). Contiguous-organ resection was needed in all grossly resected patients. IOERT was performed in 23 pts (85\%) with a median dose of 12 Gy (10-20 Gy).

We observed 7 local recurrences, transferring into estimated 3- and 5-year-LC rates of 72\%. Two were located outside the EBRT area and two were observed after more than 5 years. Locally recurrent situation had a significantly negative impact on local control. Distant failure was found in 8 pts, resulting in 3-and 5-year-DC rates of 63\%. Patients with leiomyosarcoma had a significantly increased risk of distant failure. Estimated 3- and 5-year-rates were $40 \%$ for PFS and $74 \%$ for OS. Severe acute toxicity (grade 3) was present in 4 pts (15\%). Severe postoperative complications were found in 9 pts (33\%), of whom 2 finally died after multiple re-interventions. Severe late toxicity (grade 3 ) was scored in $6 \%$ of surviving patients after 1 year and none after 2 years.

(Continued on next page)
\end{abstract}

\footnotetext{
* Correspondence: F.Roeder@dkfz.de

${ }^{1}$ Clinical Cooperation Unit Radiation Oncology, German Cancer Research

Center (DKFZ), Im Neuenheimer Feld 280, 69120 Heidelberg, Germany

2Department of Radiation Oncology, University of Heidelberg, Heidelberg,

Germany

Full list of author information is available at the end of the article
}

\section{Biomed Central}

(c) 2014 Roeder et al.; licensee BioMed Central Ltd. This is an Open Access article distributed under the terms of the Creative Commons Attribution License (http://creativecommons.org/licenses/by/4.0), which permits unrestricted use, distribution, and reproduction in any medium, provided the original work is properly credited. The Creative Commons Public Domain Dedication waiver (http://creativecommons.org/publicdomain/zero/1.0/) applies to the data made available in this article, unless otherwise stated. 
(Continued from previous page)

Conclusion: Combination of neoadjuvant IMRT, surgery and IOERT is feasible with acceptable toxicity and yields good results in terms of LC and OS in patients with high-risk retroperitoneal sarcomas. Long term follow-up seems mandatory given the observation of late recurrences. Accrual of patients will be continued with extended follow-up.

Trial registration: NCT01566123.

\section{Background}

Local control rates in patients with retroperitoneal soft tissue sarcoma (RSTS) remain disappointing even after gross total resection, mainly because wide margins are not achievable in the majority of patients [1]. In contrast to extremity sarcoma, postoperative radiation therapy (RT) has shown limited efficacy due to difficulties in achieving adequate dose and coverage [2]. Although intraoperative radiation therapy (IORT) has been introduced in some centers to overcome the dose limitations and resulted in increased outcome [3], local failure rates are still high even if considerable treatment related toxicity is accepted $[2,3]$. Compared to the postoperative approach, preoperative radiation therapy could offer several benefits, including a more precise target volume definition with smaller safety margins, reduced toxicity to adjacent organs at risk because of their displacement through the tumor itself, a possible devitalisation of tumor cells and the avoidance of treatment delays due to postoperative complications [1]. The use of Intensity-modulated radiation therapy (IMRT) further offers improved target coverage with reduced dose to adjacent organs at risk compared to conventional irradiation [4] and the opportunity to reduce overall treatment time using an integrated boost concept with simultaneously increased dose per fraction to the gross tumor volume. Since little data exists about the combination of these approaches, we initiated this prospective, nonrandomised, single center trial [5] to investigate the value of dose-escalated preoperative IMRT followed by surgery with an intraoperative electron boost to reduce the local recurrence rate without a markedly increased toxicity. Due to the slow accrual of patients, we performed an unplanned interim analysis to decide, if the trial should be continued or stopped. The results are presented here.

\section{Methods}

\section{Study design}

Details of the study design have been published elsewhere [5]. Briefly, the trial was designed as a prospective singlecenter one-armed phase I/II study. Inclusion and exclusion criteria are listed in Table 1. Pretreatment evaluation included clinical examination, laboratory tests, histological confirmation, CT or MR-imaging of the abdominal cavity, thoracic CT, bone scan, scintirenography, evaluation of general and technical resectability. For neoadjuvant IMRT, patients were immobilized using an individual body mask system or a vacuum pillow. Inverse treatment planning was based on contrast enhanced CT and MRI. The Gross Tumor Volume (GTV) included all macroscopic tumor. The clinical target volume (CTV) included the GTV with a margin of $1.5 \mathrm{~cm}$ in all directions. A safety margin of $5 \mathrm{~mm}$ was added to obtain the Planning Target Volume (PTV). Margins could be reduced with respect to anatomical borders or adjacent organs at risk. The attempted dose was 45-50 Gy to the PTV and 50-56 Gy to the GTV in 25 fractions (integrated boost concept). Doses were prescribed to the median of the GTV, while the different target volumes should be surrounded by the corresponding 95\% isodose line. An example of a dose distribution in a very large tumor is shown in Figure 1. Treatment was performed using step-and-shoot IMRT after stereotactic target point localisation. Setup correction was done using an image-guided approach with an In-Room-CT on rails at least weekly. Since October 2008 all patients have received daily In-Room-CT pretreatment setup verification and correction. Re-evaluation including restaging with abdominal CT/MRI and assessment of toxicity was scheduled 4 weeks after the last fraction of neoadjuvant irradiation. Gross total resection of the tumor was attempted within 2 weeks from restaging, including contiguous-organ or

\section{Table 1 Inclusion and exclusion criteria}

\begin{tabular}{|c|c|}
\hline Inclusion criteria & Exclusion criteria \\
\hline - Written informed consent & - Missing written informed consent \\
\hline $\begin{array}{l}\text { - Histologically confirmed, primary } \\
\text { or locally recurrent soft tissue } \\
\text { sarcoma of the retroperitoneal } \\
\text { space }\end{array}$ & $\begin{array}{l}\text { - Missing histological conformation } \\
\text { of soft tissue sarcoma }\end{array}$ \\
\hline $\begin{array}{l}\text { - Judged as at least marginally } \\
\text { resectable }\end{array}$ & $\begin{array}{l}\text { - Desmoid Tumors (aggressive } \\
\text { fibromatosis), Gastrointestinal } \\
\text { Stroma Tumors (GIST) }\end{array}$ \\
\hline - Absence of distant metastases & $\begin{array}{l}\text { - Judged as gross incomplete or not } \\
\text { resectable }\end{array}$ \\
\hline \multirow[t]{5}{*}{ - Tumor size $\geq 5 \mathrm{~cm}$} & - Incomplete staging \\
\hline & - Presence of distant metastases \\
\hline & $\begin{array}{l}\text { - Prior radiation therapy to the } \\
\text { abdominal region }\end{array}$ \\
\hline & $\begin{array}{l}\text { - Participation in another clinical } \\
\text { interventional study }\end{array}$ \\
\hline & - Inflammatory bowel disease \\
\hline
\end{tabular}




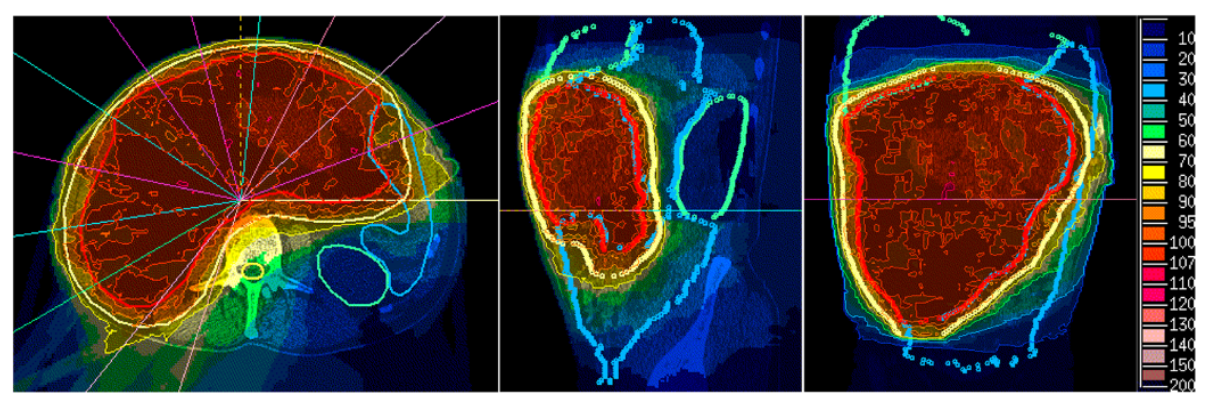

Figure 1 Example for a dose distribution in a large retroperitoneal sarcoma. Left: axial view, middle: sagittal view, right: frontal view, red line: GTV, yellow line: PTV, other coloured lines: organs at risk, legend: percentage of prescribed dose.

major vessel resection and reconstruction if necessary. IORT using a dedicated linear accelerator was directed to the whole tumor bed with an attempted dose of 10-12 Gy prescribed to the $90 \%$ isodose. If the complete tumor bed could not be covered due to its large size, IORT was directed to the intraoperatively defined high-risk region for positive margins. Definition of the high-risk region was done by the treating surgeon together with the radiation oncologist based on macroscopic evaluation of the resected specimen in correlation with the preoperative imaging. Frozen sections of the tumor bed were generally available but not routinely used. Multiple field IORT was considered and performed only if an overlap of the fields could definitively be avoided. If no coverable high risk region could be defined, IORT was not performed. Regular follow-up visits took place every 3 months after surgery for the first 2 years and every 6 months for three further years. They included at least clinical and laboratory examinations, CT or MR-imaging of the abdominal cavity and thoracic CT (every second visit).

\section{Statistical and legal considerations}

The primary objective was the local control rate after 5 years. Secondary objectives were progression-free survival, overall survival, acute and late toxicity, resectability and patterns of recurrence. Local control was defined as absence of disease progression/recurrence in the abdominal cavity (except organ metastases or diffuse peritoneal sarcomatosis). Progression-free survival (PFS) was determined as absence of local or distant recurrence/progression or death of any cause. Time to event data was calculated from the first day of radiation treatment using the Kaplan-Meier method. Acute and late radiation toxicity was assessed according to Common Terminology Criteria for Adverse Events Version 3.0 (CTCAE 3.0). Postoperative complications were assessed according to the Clavien-Dindo classification [6]. Based on the initial statistical considerations, the calculated sample size was 37 patients in the per protocol population to detect an improvement in the 5-year local control rate from 50\% (assumption from the literature after surgery and postoperative 3D-conformal radiation therapy) to $70 \%$ with a power of $80 \%$. According to the protocol, data should be analysed separately by per protocol and full set analysis population (defined as all patients who started radiation therapy). The study protocol was approved by the independent ethics committee of the Medical Faculty at the University of Heidelberg. The trial was carried out by adhering to local legal and regulatory requirements. The study complies with the Declaration of Helsinki 2004, the principles of Good clinical practice (GCP) and the German Federal Data Protection Act. Written informed consent was obtained from each patient prior to study entry.

\section{Patient characteristics}

The current interim analysis was based on the full set analysis population after the inclusion of 27 patients between 2007 and 2013. Median age at inclusion was 60 years (range $37-76$ years) and $52 \%$ of the patients were male. The majority of patients showed high-grade lesions (FNCLCC G1:18\%, G2:52\%, G3:30\%). The predominant histology was liposarcoma (70\%). Median tumor size was $15 \mathrm{~cm}(6-31 \mathrm{~cm})$. For detailed patient characteristics see Table 2 .

\section{Results}

\section{Completion of planned treatment}

The median follow-up was 33 months (5-75 months) for the entire cohort, and 37 months (8-75 months) in surviving patients. Neoadjuvant IMRT was completed as planned in 25 patients $(93 \%)$ without treatment breaks $>4$ days. In two patients neoadjuvant IMRT was prematurely finished. One suffered from local disease progression and proceeded to immediate surgery after 13 fractions, one developed grade 3 leukopenia and treatment was stopped after 23 fractions to avoid compromise of surgical treatment.

The median GTV and PTV volumes were $1146 \mathrm{ccm}$ (range 62 to $6763 \mathrm{ccm}$ ) and $2452 \mathrm{ccm}$ (range 388 to 8516 $\mathrm{ccm}$ ), respectively. A median number of 9 beams (range 5-14) with 144 segments (43 to 242) was used to deliver 
Table 2 Patient characteristics

\begin{tabular}{|c|c|c|c|}
\hline Patient characteristics & $\mathbf{n}$ & & $\%$ \\
\hline \multicolumn{4}{|l|}{ Age [yrs] } \\
\hline Median & & 60 & \\
\hline Min & & 37 & \\
\hline Max & & 76 & \\
\hline \multicolumn{4}{|l|}{ Gender } \\
\hline Male & 14 & & 52 \\
\hline Female & 13 & & 48 \\
\hline \multicolumn{4}{|l|}{ Situation } \\
\hline Primary & 23 & & 85 \\
\hline Recurrent & 4 & & 15 \\
\hline \multicolumn{4}{|l|}{ Histology } \\
\hline Liposarcoma & 19 & & 70 \\
\hline Leiomyosarcoma & 8 & & 30 \\
\hline \multicolumn{4}{|l|}{ Grading (FNCLCC) } \\
\hline G1 & 5 & & 18 \\
\hline G2 & 14 & & 52 \\
\hline G3 & 8 & & 30 \\
\hline \multicolumn{4}{|l|}{ Size $[\mathrm{cm}]$} \\
\hline Median & & 15 & \\
\hline Min & & 6 & \\
\hline Max & & 31 & \\
\hline \multicolumn{4}{|c|}{ Symptoms at presentation } \\
\hline None & 4 & & 15 \\
\hline Max grade 1 & 15 & & 55 \\
\hline Max grade 2 & 7 & & 26 \\
\hline Max grade 3 & 1 & & 4 \\
\hline
\end{tabular}

Centres de Lutte Contre le Cancer, cm: centimeter.

median doses of 50 Gy (range 26-55 Gy) to the GTV and 45 Gy (23.4 to 50 Gy) to the PTV (see Table 3).

The median time spans from the end of neoadjuvant radiation treatment to restaging and from restaging to surgery were 28 days (12-38 days) and 11 days (1-33 days), respectively (excluding the patient with immediate surgery due to progression who did not receive formal restaging). The median time from the end of neoadjuvant radiation treatment until surgery was 39 days (range 6-62 days).

Gross total resection was achieved as planned in 26 patients (96\%). Final resection margin was R0 in 6 patients (22\%) and R1 in 22 patients (74\%). One patient was judged non-resectable intraoperatively. Contiguous organ resection to achieve grossly free margins was required in all resected patients (see Table 3). Major vessel resection and reconstruction was needed in 8 patients (30\%). Major nerve resections were required in 2 patients and partial resection of the psoas muscle or diaphragm was necessary in 9 patients (see Table 3).

IORT was performed in 23 patients $(85 \%)$ including the non-resectable patient. One patient received no IORT due to technical reasons and in the remaining three patients a coverable high-risk region could not be defined intraoperatively. The median IORT dose was 12 Gy (range 10-20 Gy) with a median electron energy of $8 \mathrm{MeV}$ (range 6-12 MeV) and a median cone size of $8 \mathrm{~cm}$ (range $5-18 \mathrm{~cm}$ ), see Table 3 .

\section{Oncological endpoints}

We found seven local recurrences (26\%), transferring into estimated 3- and 5-year local control rates of $72 \%$, respectively (Figure 2). Of note, two of the recurrences were located near but outside the EBRT areas, two were observed after more than 5 years of follow-up and three were found in patients with recurrent situation at inclusion. Recurrent situation at inclusion was the only factor with significantly negative impact on local control in univariate analysis. Patients presenting in primary situation showed estimated 3- and 5-year local control rates of $88 \%$. Local failures were treated by surgery (with or without IOERT) including histological confirmation in 6 patients and with palliative systemic treatment without histological confirmation in one patient with simultaneously diagnosed diffuse metastatic disease. Of the two recurrences observed after more than 5 years, one was histologically confirmed and salvaged by surgery.

Distant failures were observed in eight patients (30\%), transferring into estimated 3- and 5-year distant control rates of $63 \%$, respectively (Figure 3 ). Seven of them occurred during the first two years of follow-up. Histology of leiomyosarcoma was the only factor with significantly negative impact on distant control in univariate analysis.

The 3- and 5-year progression-free survival rates were $40 \%$, respectively. Of the 16 observed events, 5 were isolated local recurrences, 6 were isolated distant failures, 2 were combined failures and 3 were deaths without disease progression. Histology of leiomyosarcoma was the only factor with significantly negative impact on progressionfree survival. Furthermore we found a trend for improved PFS in primary vs. recurrent situation.

Six patients died during follow-up, transferring into estimated 3- and 5-year overall survival rates of $74 \%$ (Figure 4), respectively. None of the tested factors showed a significant impact on overall survival.

\section{Toxicity}

Acute radiation related toxicity was mainly hematological and gastrointestinal. The maximum acute toxicity was grade 1 in 10 patients (37\%), grade 2 in 13 (48\%) patients and grade 3 in 4 patients (15\%). For detailed analysis see Table 4. 
Table 3 Treatment characteristics

\begin{tabular}{|c|c|c|c|c|}
\hline Treatment & n $\%$ & & $\mathrm{n}$ & $\%$ \\
\hline Neoadjuvant IMRT & & Surgery & & \\
\hline Completed & 2593 & Gross total & 26 & 96 \\
\hline Not completed & 2 & Explo. lap & 1 & 4 \\
\hline GTV volume [ccm] & & Resection margin & & \\
\hline Median & 1146 & Ro & 6 & 22 \\
\hline Min & 62 & $\mathrm{R} 1$ & 20 & 74 \\
\hline Max & 6763 & Explo. Lap. & 1 & 4 \\
\hline PTV volume [ccm] & & Cont. organ resection & & \\
\hline Median & 2452 & yes & 26 & 96 \\
\hline Min & 388 & no & 1 & 4 \\
\hline \multirow[t]{2}{*}{ Max } & 8516 & & & \\
\hline & & Number of organs* & & \\
\hline GTV dose [Gy] & & 0 (explo Lap) & 1 & 4 \\
\hline Median & 50 & 1 & 7 & 26 \\
\hline Min & 26 & 2 & 8 & 30 \\
\hline \multirow[t]{2}{*}{ Max } & 55 & 3 & 5 & 19 \\
\hline & & 4 & 6 & 22 \\
\hline
\end{tabular}

PTV dose [Gy]

\begin{tabular}{|c|c|c|c|c|}
\hline Median & 45 & Type of organ & & \\
\hline Min & 23,4 & Nephrectomy & 18 & 67 \\
\hline \multirow[t]{2}{*}{ Max } & 50 & Hemicolectomy & 16 & 59 \\
\hline & & Splenectomy & 8 & 30 \\
\hline Number of beams & & Partial pancreatectomy & 8 & 30 \\
\hline Median & 9 & Cholecystectomy & 8 & 30 \\
\hline Min & 5 & Small bowel resection & 3 & 11 \\
\hline \multirow[t]{2}{*}{ Max } & 14 & Rectum resection & 3 & 11 \\
\hline & & Partial colpectomy & 2 & 7 \\
\hline Number of segments & & Partial liver resection & 1 & 4 \\
\hline Median & 144 & Cystectomy & 1 & 4 \\
\hline Min & 43 & Adnexectomy & 1 & 4 \\
\hline Max & 242 & Hysterectomy & 1 & 4 \\
\hline IOERT & & Muscle resection & & \\
\hline Completed & 23 & Yes & 9 & 33 \\
\hline \multirow[t]{2}{*}{ Not completed } & 4 & No & 18 & 67 \\
\hline & & Psoas & 4 & 15 \\
\hline IOERT dose [Gy] & & Diaphragm & 6 & 22 \\
\hline Median & 12 & & & \\
\hline Min & 10 & Vessel resection & & \\
\hline \multirow[t]{2}{*}{ Max } & 20 & Yes & 8 & 30 \\
\hline & & No & 19 & 70 \\
\hline IOERT energy [MeV] & & Major artery & 4 & 15 \\
\hline Median & 8 & Major vein & 7 & 26 \\
\hline Min & 6 & & & \\
\hline Max & 12 & Nerve resection & & \\
\hline
\end{tabular}

Table 3 Treatment characteristics (Continued)

\begin{tabular}{lccc}
\hline & Yes & 2 & 7 \\
IOERT cone size [cm] & No & 25 & 93 \\
Median & 8 & & \\
Min & 5 & $*$ \\
Max & 18 & \\
\hline n:number of patients, \%: percentage, IMRT: intensity-modulated radiation \\
therapy, GTV: gross tumor volume, PTV: planning target volume, ccm : cubic \\
centimetre, Gy: gray, IOERT : intraoperative electron radiation therapy, MeV : \\
mega electron volts, cm : centimetre, explo. Lap. : explorative laparotomy, R0: \\
microscopically negative, R1 : microscopically positive, cont. contiguous.
\end{tabular}

Severe postoperative complications included bowel/anastomotic leakage, pancreatic fistula/leakage, intra-abdominal bleeding, sepsis and left ventricular dysfunction. The 30day postoperative mortality rate was $0 \%$, but 2 patients finally died in the prolonged postoperative period. The maximum postoperatively observed toxicity was grade 1 in 7 patients (26\%), grade 2 in 10 patients (37\%), grade 3a in 5 patients (18\%), grade $3 \mathrm{~b}$ in 1 patient (4\%), grade $4 \mathrm{~b}$ in 1 patient (4\%) and grade 5 in 2 patients (7\%). 4 patients (15\%) needed re-laparotomies (2 of them multiple ones). One of the deceased patients developed a pancreatic/duodenal leakage after partial pancreatic and major vessel resection, but initially denied re-laparotomy and finally died due to massive intra-abdominal bleeding 3 months later although emergency surgery was performed. The other patient developed a pancreatic and multiple bowel leakages with intraabdominal bleeding complications after multivisceral resection including partial pancreatectomy, nephrectomy, hemicolectomy and splenectomy, and finally died after multiple re-interventions due to septic complications. For detailed analysis see Table 5.

Observed late toxicity was mainly gastrointestinal, genitourinary and neurological, but generally mild. As late toxicity may vary over time, it was analysed separately at different time points. Patients with salvage treatments were excluded from analysis. Suitable information was available in 16 patients at one year and 14 patients at two years. Maximum toxicity at one year was grade 0 in 4 patients $(25 \%)$, grade 1 in 7 patients (44\%), grade 2 in 4 patients (25\%) and grade 3 in one patient $(6 \%)$. Maximum toxicity at two years was grade 0 in 4 patients (29\%), grade 1 in 7 patients (50\%) and grade 2 in 5 patients (36\%). For detailed analysis see Table 6.

\section{Discussion and conclusions}

The optimal management of retroperitoneal soft tissue sarcomas including the role of radiation therapy has been extensively debated in the past and remains still unclear. In contrast to extremity sarcoma, data from large randomized trials regarding the optimal treatment for retroperitoneal sarcoma is still lacking due to the rarity of this disease and even prospective phase I/II data is rare. 


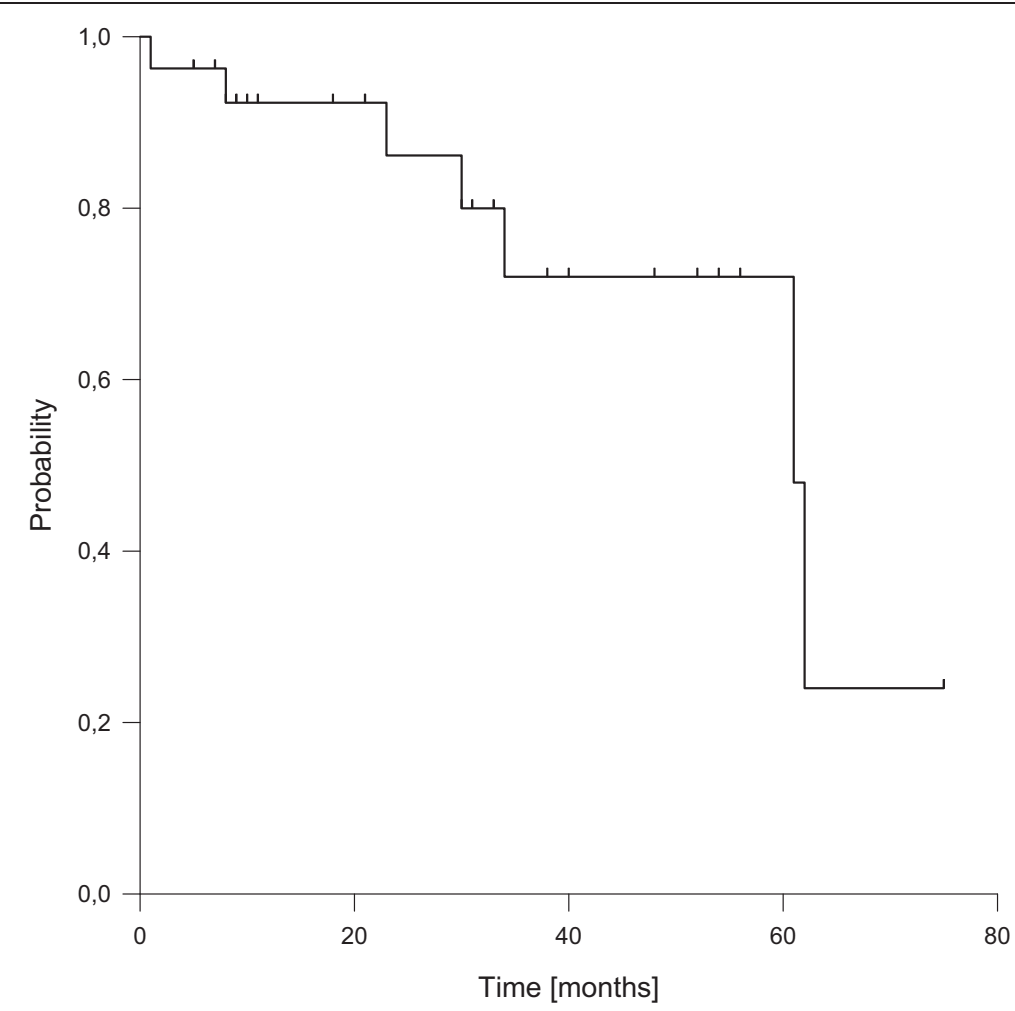

Figure $\mathbf{2}$ Local control.

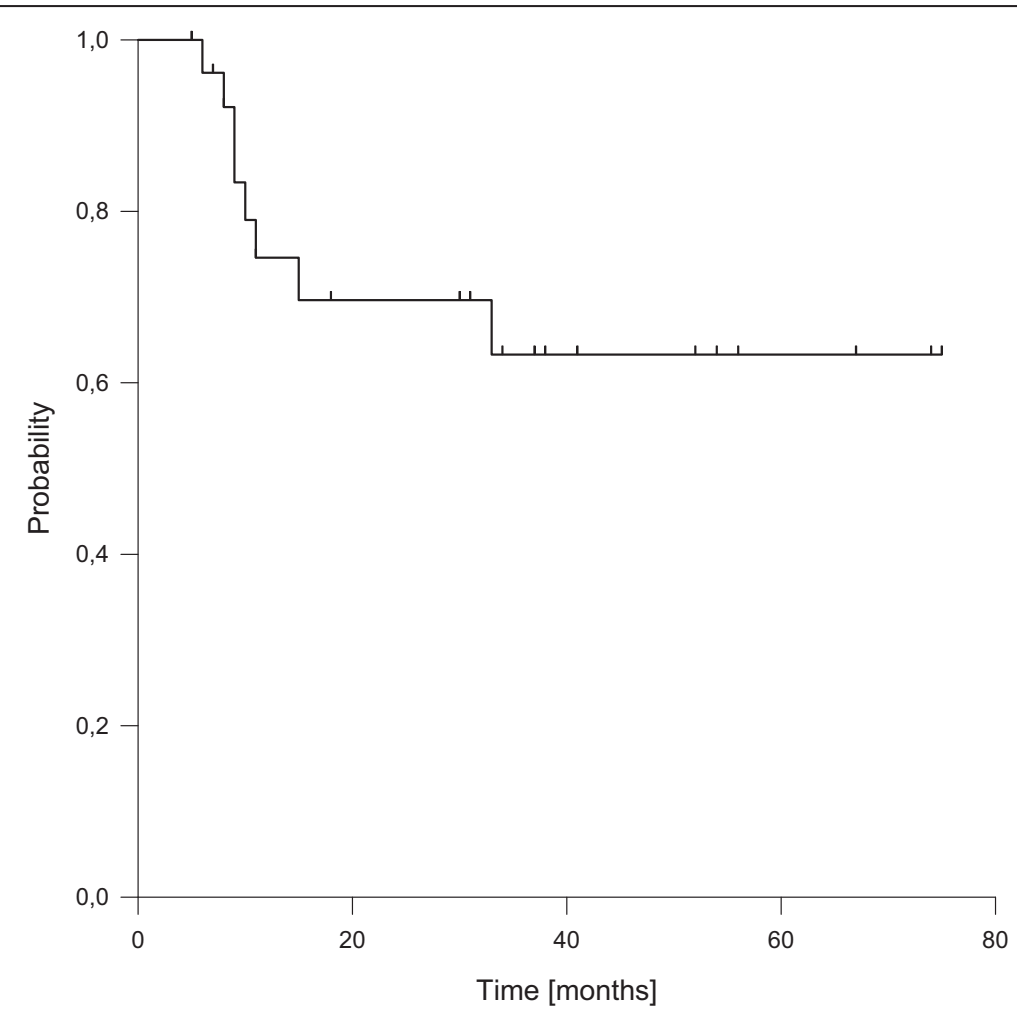

Figure 3 Distant control. 


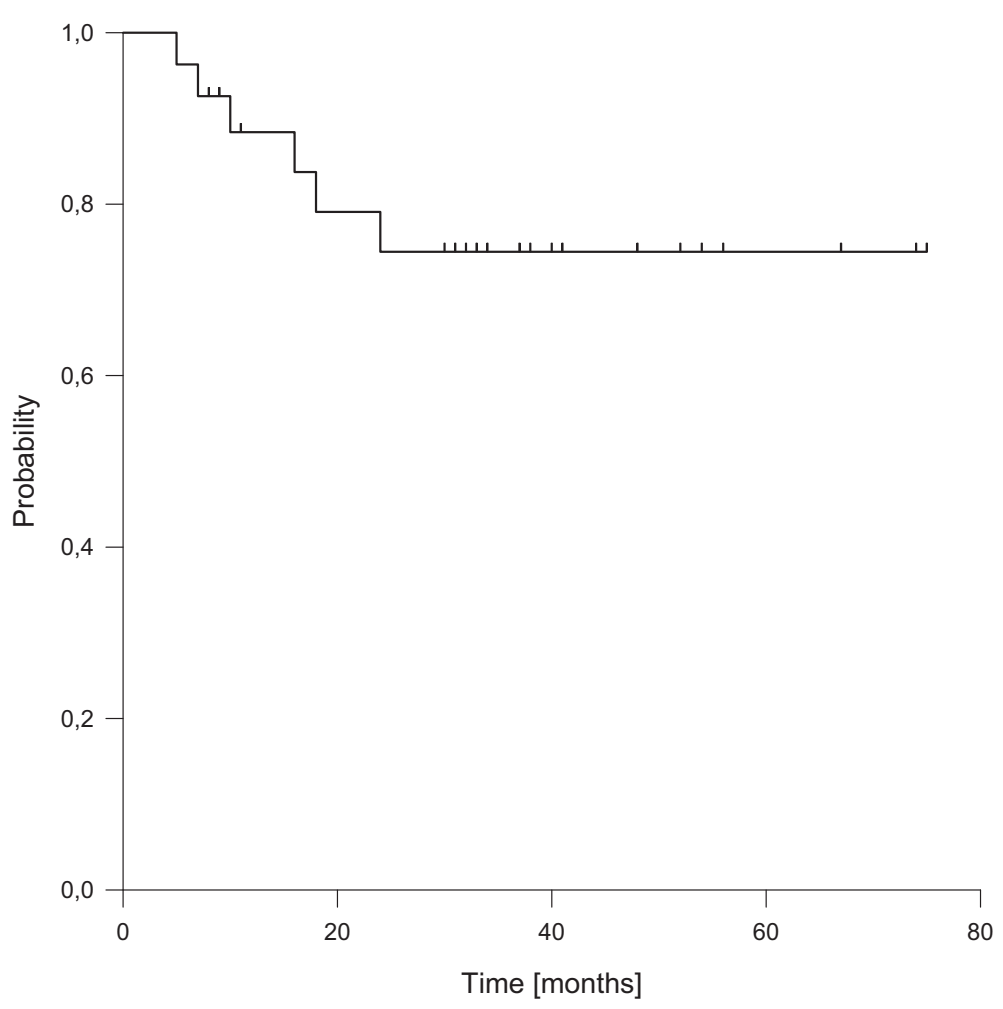

Figure 4 Overall survival.

Treatment recommendations are mainly based on retrospective single center series, usually covering small patients numbers treated over many years with various combinations of surgical approaches and radiation treatment modalities. Therefore, evidence gained in the much larger studies in extremity sarcoma patients is frequently transferred to guide treatment of retroperitoneal sarcomas. As margin status has been identified as an important prognostic factor in both extremity and retroperitoneal sarcomas $[7,8]$ surgery with wide negative margins represents the cornerstone of curative intent approaches. However, the achievement of wide or even close negative margins is much more difficult in the retroperitoneal space than in the extremities, resulting in much higher local recurrence rates after surgery alone [9]. Even with more aggressive surgical approaches using en bloc resections of adjacent uninvolved organs, local recurrence remains the dominant pattern of failure in retroperitoneal sarcomas $[10,11]$. Additional radiation therapy has clearly been shown to improve local control in extremity sarcomas irrespective of margin status with increasing benefits after close or positive margin resections [7] and is therefore widely accepted as standard of care for these patients. As close or positive margins are more frequent in retroperitoneal sarcomas, this should theoretically lead to an even more pronounced benefit from additional radiation therapy, but only a minority of patients is currently treated with this combination approach [12]. Postoperative external beam radiation therapy was the first RT modality, which has been investigated. But although local control seemed to be improved in many series compared to surgery alone $[11,13,14]$, concerns have been raised mainly by the difficulties in achieving adequate dose and target coverage in the postoperative setting. Delivery of efficient doses with generous margins as used in extremity sarcomas is often hampered by the presence of small bowel loops in the resection cavity which would result in unacceptable toxicity in many cases, while reduced doses or margins would compromise efficacy. Because of the known dose-effect relationship which favours doses $\geq 55$ Gy $[15,16]$, several institutions including ours investigated additional boosting techniques like intraoperative radiation therapy (IORT) or brachytherapy to overcome these limitations $[2,3,17-19]$. The only randomized trial comparing different local treatment approaches in retroperitoneal sarcoma showed a clear benefit favouring a combination of postoperative EBRT (35-40 Gy) and IORT (20 Gy) compared to postoperative EBRT alone (50-55 Gy) in terms of local control (60\% vs. $20 \%)$ and gastrointestinal toxicity, while neurological toxicity was markedly increased in the IORT arm [3]. Several non-randomized single institution series have confirmed high rates of local control for this combination approach with acceptable neurological toxicities limiting the IORT dose to 15 Gy $[2,17,18,20]$, but 
Table 4 Acute toxicity (including the preoperative period)

\begin{tabular}{|c|c|c|c|c|}
\hline Toxicity & All grades & Grade 1 & Grade 2 & Grade 3 \\
\hline \multicolumn{5}{|l|}{ GI } \\
\hline Diarrhea & 11 & 5 & 4 & 2 \\
\hline Nausea & 11 & 6 & 5 & 0 \\
\hline Appetite loss & 4 & 3 & 1 & 0 \\
\hline Bloating & 8 & 3 & 5 & 0 \\
\hline Reflux & 3 & 2 & 1 & 0 \\
\hline Constipation & 3 & 1 & 2 & 0 \\
\hline \multicolumn{5}{|l|}{ Hematological } \\
\hline Anemia & 18 & 12 & 5 & 1 \\
\hline Leucopenia & 9 & 5 & 3 & 1 \\
\hline Thrombopenia & 8 & 8 & 0 & 0 \\
\hline \multicolumn{5}{|l|}{ Skin } \\
\hline Erythema & 10 & 10 & 0 & 0 \\
\hline \multicolumn{5}{|l|}{ GU } \\
\hline Frequency/urgency & 3 & 1 & 2 & 0 \\
\hline Dysuria & 4 & 3 & 1 & 0 \\
\hline Bladder obstruction & 1 & 0 & 1 & 0 \\
\hline Ureter obstruction & 1 & 0 & 0 & 1 \\
\hline Infection & 2 & 0 & 1 & 1 \\
\hline \multicolumn{5}{|l|}{ Other } \\
\hline Pain & 6 & 6 & 0 & 0 \\
\hline Fatigue & 2 & 1 & 1 & 0 \\
\hline Motor neuropathy* & 1 & 0 & 1 & 0 \\
\hline Varicocele & 1 & 0 & 1 & 0 \\
\hline Hypokalemia & 1 & 1 & 1 & 1 \\
\hline
\end{tabular}

Gl: gastrointestinal, GU: genitourinary, *:transient.

there still remains room for improvement regarding both local control and toxicity, especially if compared to extremity sarcomas. Preoperative radiation therapy offers several possible advantages compared to the postoperative approach. These include a possible sterilization of the operative field against seeding, a possible thickening of the often-present pseudocapsule easing resection and the avoidance of repopulation through treatment delays because of postoperative complications. However, the main advantage seems to be the more accurate target volume definition with the possibility of reduced safety margins and reduced toxicity especially to small bowel loops because of their displacement through the tumor itself. Furthermore, the improved oxygenation could increase radiosensitivity and lower the required dose as known from extremity sarcomas. Some of these advantages, especially regarding target coverage and reduction of dose to adjacent organs at risk, can be further exploited with the use of modern radiation techniques like IMRT, VMAT or Tomotherapy as shown in several planning
Table 5 Complications in the postoperative period

\begin{tabular}{|c|c|c|c|c|c|c|c|}
\hline Complication & All grades & 1 & 2 & $3 a$ & $3 b$ & $4 b$ & 5 \\
\hline \multicolumn{8}{|l|}{ GI } \\
\hline Functional bowel obstruction & 5 & 1 & 3 & 1 & & & \\
\hline Pancreatic fistula/leakage & 5 & & & $5^{* *}$ & & & \\
\hline Intraabdominal bleeding & 3 & & & & & 2 & 1 \\
\hline Bowel/anastomotic leakage & 2 & & & & & 1 & 1 \\
\hline Bloating/distension & 2 & 1 & 1 & & & & \\
\hline Constipation & 2 & 2 & & & & & \\
\hline Diarrhea & 2 & 2 & & & & & \\
\hline Ascites & 1 & & & 1 & & & \\
\hline Ulcus ventriculi/duodeni & 1 & & 1 & & & & \\
\hline Infection* & 2 & & 1 & & & 1 & \\
\hline \multicolumn{8}{|l|}{ Wound complication } \\
\hline Wound healing disturbance & 5 & 2 & 1 & 1 & 1 & & \\
\hline Lymph edema & 3 & 3 & & & & & \\
\hline Lymph fistula & 1 & & 1 & & & & \\
\hline Decubitus & 1 & 1 & & & & & \\
\hline Hematoma & 1 & & & & 1 & & \\
\hline \multicolumn{8}{|l|}{ Neuropathy } \\
\hline Motor neuropathy/weakness & 5 & 5 & & & & & \\
\hline Sensory neuropathy & 5 & 5 & & & & & \\
\hline \multicolumn{8}{|l|}{ GU } \\
\hline Renal failure & 5 & 5 & & & & & \\
\hline Cystitis/infection & 4 & & 4 & & & & \\
\hline Ureter obstruction & 2 & 2 & & & & & \\
\hline Urinary incontinence & 1 & 1 & & & & & \\
\hline Scrotal edema & 1 & 1 & & & & & \\
\hline
\end{tabular}

Cardiac/vascular

Av block

Ventricular dysfunction

Hypertension

Deep vein thrombosis

Pulmonary

Pleural effusion

Pneumothorax

Hematological

$\begin{array}{llll}\text { Anemia } & 2 & 1 & 1\end{array}$

Leucopenia

11

Gl: gastrointestinal, GU: genitourinary, *not leakage/fistula associated **managed with CT-guided drainage procedures.

studies [4,21-23], including the opportunity to reduce overall treatment time by an integrated boost concept.

In our present study, we therefore combined the theoretically advantageous techniques of preoperative intensitymodulated RT with an integrated boost concept, surgery and IORT with the aim to achieve maximal local control. 
Table 6 Late toxicity

\begin{tabular}{|c|c|c|c|c|c|c|c|c|}
\hline \multirow[t]{2}{*}{ Toxicity } & \multicolumn{2}{|c|}{ All grades } & \multicolumn{2}{|c|}{ Grade 1} & \multicolumn{2}{|c|}{ Grade 2} & \multicolumn{2}{|c|}{ Grade 3} \\
\hline & $1 \mathrm{yr}$ & $2 \mathrm{yrs}$ & $1 \mathrm{yr}$ & $2 \mathrm{yrs}$ & $1 \mathrm{yr}$ & $2 \mathrm{yrs}$ & $1 \mathrm{yr}$ & $2 \mathrm{yrs}$ \\
\hline \multicolumn{9}{|l|}{$\overline{\mathrm{GI}}$} \\
\hline Diarrhea & 3 & 2 & 3 & 2 & 0 & 0 & 0 & 0 \\
\hline Constipation & 1 & 2 & 1 & 2 & 0 & 0 & 0 & 0 \\
\hline Bloating & 2 & 2 & 0 & 0 & 2 & 2 & 0 & 0 \\
\hline \multicolumn{9}{|l|}{ GU } \\
\hline Frequency/urgency & 1 & 1 & 1 & 1 & 0 & 0 & 0 & 0 \\
\hline Urinary retention & 1 & 1 & 1 & 1 & 0 & 0 & 0 & 0 \\
\hline Cystitis & 1 & 0 & 1 & 0 & 0 & 0 & 0 & 0 \\
\hline \multicolumn{9}{|l|}{ Other } \\
\hline Motor neuropathy/weakness & 4 & 3 & 1 & 1 & 3 & 2 & 0 & 0 \\
\hline Sensory neuropathy & 5 & 3 & 5 & 3 & 0 & 0 & 0 & 0 \\
\hline Lymph edema & 4 & 4 & 2 & 3 & 2 & 1 & 0 & 0 \\
\hline Pain & 2 & 3 & 2 & 3 & 0 & 0 & 0 & 0 \\
\hline Spondylodiscitis & 1 & 0 & 0 & 0 & 0 & 0 & 1 & 0 \\
\hline
\end{tabular}

Gl: gastrointestinal, GU.genitourinary, yr: year, yrs: years.

Unfortunately, we faced the same difficulties as many other groups investigating this rare disease, namely poor accrual over a long time span. We therefore decided to perform this unplanned interim analysis to evaluate whether the initial aims of the study are still reasonably achievable. With a median follow-up of 33 months, we found encouraging local control and overall survival rates (estimated 5-year LC 72\%, and 5-year OS 74\%), especially given the unfavourable patient selection (median tumor volume $1146 \mathrm{ccm}$ and surgery with negative margins possible in only $22 \%$ of the patients). Moreover, we found a change in the pattern of failure, with distant metastasis being more prevalent than local recurrences. However, two recurrences were observed shortly after 5 years of followup, indicating the need for longer follow up to draw definitive conclusions. Nevertheless, our preliminary results seem to compare favourably with other groups using similar approaches. Pawlik et al. [1] reported a combined analysis of two prospective trials including 72 patients with high grade retroperitoneal sarcomas treated with preoperative radiation therapy and surgery. Preoperative radiation was completed in $89 \%$ of the patients and 57 proceeded to surgery. Gross total resection was achieved in 54 patients of whom 32 received an additional boost via IORT or brachytherapy. With a median follow-up of 40 months, they observed 2- and 5-year local control rates of $79 \%$ and $60 \%$ and a 5 -year overall survival rate of $61 \%$ after gross total resection. Gronchi et al. [24] reported a prospective trial of 83 patients, who were treated by preoperative radiation therapy combined with chemotherapy. Radiation therapy was completed as planned in 73 and an additional IORT boost was given in 14 patients. 79 patients
(95\%) underwent surgery. With a median f/u of 58 months, 5-year local control, distant control and overall survival rates were $63 \%, 74 \%$ and $59 \%$, respectively. Smith et al. [25] published the long term analysis of a prospective trial which investigated preoperative radiation in 40 patients combined with selectively applied postoperative brachytherapy. After a median follow-up of 106 months, 5-year overall survival was reported to be $70 \%$ with a crude local control rate of $68 \%$. Tzeng et al. [26] reported a small prospective trial investigating dose escalated preoperative IMRT with integrated boost in 16 patients. Gross total resection was achieved in 14 patients, resulting in a 2-year local control rate of $80 \%$ after a median $\mathrm{f} / \mathrm{u}$ of 28 months. These results were also supported by several retrospective series which reported 5-year local control rates of $63-68 \%$ and 5-year overall survival rates of $64-72 \%$ with similar approaches [27-29], see Table 7. In summary, preoperative radiation therapy followed by surgery with or without additional boost consistently reached high local control and overall survival rates, which seem to be superior to the results of surgery alone or combinations of surgery with postoperative radiation, although formal high level evidence is still lacking. Therefore two phase III trials (ACOSOG Z9031, EORTC 62092) have been designed to evaluate preoperative radiation. While the first trial has already been closed due to poor accrual [27], the results of the ongoing EORTC trial are eagerly awaited and will hopefully clarify the role of preoperative radiation therapy.

Besides oncological outcome, every additional therapy comes along with toxicity. Therefore, the possible benefits in terms of local control or overall survival have to be weighed against side effects. In our study, mild gastrointestinal and 
Table 7 Series with preoperative radiation therapy

\begin{tabular}{|c|c|c|c|c|c|c|c|c|c|}
\hline Author & Year & Type & $n$ & $f / u$ & Pre RT & GTR & Boost & 5-year-LC & 5-year-OS \\
\hline$\overline{\text { Pawlik' }}$ & 2006 & Pro.,comb. & 72 & 40 & $89 \%$ & $79 \%$ & $44 \%$ & $60 \% *$ & $61 \%^{*}$ \\
\hline Gronchi $^{24}$ & 2014 & Pro. & 83 & 58 & $88 \%$ & $95 \%$ & $17 \%$ & $63 \%$ & $59 \%$ \\
\hline Smith $^{25}$ & 2014 & Pro.,subgr. & 40 & 106 & $100 \%$ & $100 \%$ & $48 \%$ & $63 \%(c r)$ & $70 \%$ \\
\hline Tzeng ${ }^{26}$ & 2006 & Pro. & 16 & 28 & $100 \%$ & $88 \%$ & $0 \%$ (d) & $80 \%(2 \mathrm{yr})$ & n.s. \\
\hline McBride $^{27}$ & 2013 & Retro. & 33 & 33 & $100 \%$ & $100 \%$ & $30 \%$ & $63 \%(3 \mathrm{yr})$ & $64 \%$ (3 yr) \\
\hline Sweeting ${ }^{28}$ & 2013 & Retro. & 18 & 43 & $94 \%$ & $100 \%$ & $100 \%$ & $64 \%$ & $72 \%$ \\
\hline Alford $^{29}$ & 2012 & Retro. & 24 & 28 & $100 \%$ & $75 \%$ & $0 \%$ & $68 \%$ & $54 \%$ \\
\hline Present data & 2014 & Pro.,interim & 27 & 33 & $93 \%$ & $96 \%$ & $85 \%$ & $72 \%$ & $74 \%$ \\
\hline
\end{tabular}

pro.: prospective trial, comb., combined analysis, subgr.: subgroup analysis, interim: interims analysis, $\mathrm{n}$ : number of patients, $\mathrm{f} / \mathrm{u}$ : median follow-up in months, pre RT: percentage of patients with completion of preoperative radiation therapy as planned, GTR: percentage of patients in whom gross total resection was achieved, boost: percentage of patients who received an additional boost via IORT or Brachytherapy, d: preoperative radiation therapy was dose escalated, LC: local control, OS: Overall survival, *: in grossly resected patients, cr: crude rate, 2 yr: 2-year rate, 3 yr: 3-year rate.

hematological toxicity was common during preoperative radiation therapy, but only 4 patients (15\%) suffered from grade III acute toxicity, which seems quite acceptable given the large radiation fields (median tumor size $15 \mathrm{~cm}$, median PTV $2400 \mathrm{ccm}$ ). Jones et al. [30] described GI/pelvic grade I toxicity in $34 \%$ and grade II in $47 \%$ with no grade III side effects in their prospective trial on preoperative radiation. Pisters et al. [31] reported a dose escalation trial with simultaneously given doxorubicin and described high rates of acute gastrointestinal grade III-IV toxicity (18\%) and hematological toxicity $(27 \%)$ in patients treated at the 50.4 Gy dose level, although at least parts of the side effects might be attributable to chemotherapy. Gronchi et al. [24] also reported higher grade III/IV toxicity rates, but again this trial included simultaneously applied chemotherapy and therefore is difficult to compare. Caudle et al. [32] found acute toxicity in $43 \%$ of 14 patients treated with preoperative radiation. From the description, it can be estimated that the rate of severe acute side effects (grade 3 or higher) was $21 \%$, although toxicity was not formally graded. Zlotecki et al. [33] compared patients with preoperative and postoperative radiation and found significantly decreased severe acute side effects after preoperative radiation therapy (36\% vs 80\%).

Preoperative radiation did not compromise the general ability for surgery in our study, as all patients proceeded to surgery and all except one received gross total resection. However, we observed a considerable rate $(33 \%)$ of severe postoperative complications, including 2 patients (7\%) who finally died after multipleinterventions in the prolonged postoperative period (30 day postoperative mortality rate was $0 \%$ ). Jones et al. [30] reported severe postoperative complications in $41 \%$ of their patients treated with preoperative radiation and selectively applied brachytherapy. They further described a 30 day mortality rate of $2 \%$, but two additional patients (4\%) died during the following 18 months due to anastomotic leakage and duodenal perforation. Gronchi et al.
[24] observed 21\% major postoperative complications after preoperative chemoradiation and Alford et al. [29] found $44 \%$ severe postoperative complications after preoperative radiation without additional boosting techniques. These results raise the question, if preoperative radiation increases the postoperative complication rate. However, this question is difficult to answer, as data from prospective series using surgery only is rare. Strauss et al. [34] described a 30 day mortality rate of $3 \%$ after surgery alone. Lewis et al. [35] reported a 30 day mortality rate of $4 \%$ in a large series of patients treated with surgery only or additional radiation and chemotherapy. Bonvalot et al. reported a mortality rate of $3 \%$ with only $18 \%$ of their patients requiring an invasive therapeutic procedure and $12 \%$ re-operation rate in a series treated with so called "aggressive frontline surgery" [36]. About one third of the patients had received preoperative radiation, but unfortunately the complication rate was not reported separately for the groups with or without radiation treatment. Zlotecki et al. [33] compared pre- and postoperative radiation therapy and observed a significant difference in severe postoperative morbidity ( $20 \%$ vs $53 \%$ ) favouring the preoperative approach. Finally, Bartlett et al. [37] compared preoperative radiation with surgery alone in a large retrospective series and found no significant differences, neither in overall morbidity and mortality nor in specific side effects. Given the extended surgical approach with contiguous organ resection in our patients, the postoperative complication rate seems acceptable and at least not distinctly increased by preoperative radiation. However, regarding the differences in mortality rates covering different postoperative time spans and the wide range of "severe" postoperative complications reported in the (mainly retrospective) literature after surgery with or without radiation therapy, further clarification is needed by standardized reporting of toxicity data from prospective trials.

Severe late toxicity was uncommon in our study, as only 1 patient (6\%) showed grade III late toxicity at one 
year and none of the patients at two years. Jones et al. [30] reported a $10 \%$ severe late toxicity rate at 18 months and Smith et al. [25] described severe late toxicity in $11 \%$ of the patients after 18 months in an updated report of the same trial with mature follow-up. Alford et al. [29] observed late toxicities of any grade in $46 \%$ of their patients. Interestingly, they described neurological lower limb side effects in $21 \%$, although no additional boost via IORT has been performed, indicating that at least parts of the neuropathy effects usually attributed to IORT treatments might be caused by other reasons. However, the low rate of neuropathy might been explained by the lower IORT doses used in our trial compared to many other series. Based on the early findings reported by Shaw et al., which described a significant association between increased IORT dose and neuropathy rate [20], the IORT dose was limited to 10-12 Gy in our study protocol. Interestingly, Haddock et al. described a significant reduction of neuropathy with intraoperative doses of less than 12.5 Gy in a recently updated series of rectal cancer treated with IORT [38], indicating that our dose constraint seemed reasonable.

Clearly our analysis has some limitations mainly because of its nature of an unplanned interim analysis due to slow accrual. This naturally results in a relatively small number of patients with short follow-up, thus limiting the ability to draw definitive conclusions. Nevertheless, it represents prospectively collected data for the combination of preoperative radiation therapy using IMRT followed by aggressive surgery with contiguous organ resection and intraoperative radiation therapy in this rare disease of retroperitoneal sarcoma and therefore adds valuable information to the small existing body of evidence for this combination approach.

In summary, combination of preoperative IMRT, surgery and IORT resulted in promising 5-year local control and overall survival rates with low rates of acute and late toxicity and acceptable postoperative complications. Long term follow-up seems mandatory given the observation of late recurrences. Accrual of patients will be continued with extended follow-up.

\section{Competing interests}

The authors declare that they have no competing interests.

\section{Authors' contributions}

FR drafted the manuscript, participated in data aquisition and statistical analysis and supervised preoperative and intraoperative radiation therapy. $\mathrm{AU}$ and IA participated in data acquisition and treated the patients surgically. $\mathrm{GH}$ and MU participated in data acquisition and intraoperative radiation treatment. LSE participated in preoperative radiation treatment, data acquisition, statistical analysis and preparation of the manuscript. PEH participated in conducting the study, preparing the manuscript and reviewed the manuscript critically. FWH assisted in study conception, preparation of the study protocol and participated in conducting the trial regarding the aspects of radiation physics. DSE planned the study, prepared the study protocol, conducted the correspondence with the legal authorities and participated in study conduction. AN assisted in planning of the study, preparation of the study protocol and participated in conducting the study. RK supervised intraoperative radiation therapy, and participated in data acquisition. GM participated in conducting the trial regarding the aspects of pathology. JD participated in study conception and design. MB participated in manuscript preparation, conduction of the study and reviewed the manuscript critically. All authors read and approved the final manuscript.

\section{Acknowledgements}

The study was an investigator initiated trial (IIT) funded by the University of Heidelberg.

\section{Author details}

${ }^{1}$ Clinical Cooperation Unit Radiation Oncology, German Cancer Research Center (DKFZ), Im Neuenheimer Feld 280, 69120 Heidelberg, Germany. ${ }^{2}$ Department of Radiation Oncology, University of Heidelberg, Heidelberg, Germany. ${ }^{3}$ Department of Surgery, University of Heidelberg, Heidelberg, Germany. ${ }^{4}$ Department of Radiation Oncology, Markus Clinic, Frankfurt, Germany. ${ }^{5}$ Department of Radiation Oncology, Helios Clinic, Berlin-Buch, Germany. ${ }^{6}$ Institute of Pathology, University of Heidelberg, Heidelberg, Germany. ${ }^{7}$ Department of Radiation Oncology, SLK Clinic, Heilbronn, Germany. ${ }^{8}$ Department of Radiation Oncology, University of Munich (LMU), Munich, Germany.

Received: 20 May 2014 Accepted: 20 August 2014

Published: 27 August 2014

\section{References}

1. Pawlik TM, Pisters PW, Mikula L, Feig B, Hunt KK, Cormiert JN, Ballo MT, Catton CN, Jones JJ, O'Sullivan B, Pollock RE, Swallow CJ: Long-term results of two prospective trials of preoperative external beam radiotherapy for localized intermediate- or high-grade retroperitoneal soft tissue sarcoma. Ann Surg Oncol 2006, 13:508-517.

2. Krempien R, Roeder F, Oertel S, Weitz J, Hensley FW, Timke C, Funk A, Lindel K, Harms W, Buchler MW, Debus J, Treiber M: Intraoperative electron-beam therapy for primary and recurrent retroperitoneal soft-tissue sarcoma. Int J Radiat Biol Oncol Phys 2006, 65:773-779.

3. Sindelar WF, Kinsella TJ, Chen PW, DeLaney TF, Tepper JE, Rosenberg SA, Glatstein E: Intraoperative radiotherapy in retroperitoneal sarcomas: final results of a prospective, randomized clinical trial. Arch Surg 1993, 128:402-410.

4. Musat E, Kantor G, Caron J, Lagarde P, Laharie H, Stoeckle E, Angles J, Gilbeau L, Bui BN: Comparison of intensity-modulated postoperative radiotherapy with conventional postoperative conformal radiotherapy for retroperitoneal sarcoma. Cancer Radiother 2004, 8:255-261.

5. Roeder FF, Schulz-Ertner D, Nikoghosyan AV, Huber PE, Edler L, Habl G, Krempien R, Oertel S, Saleh-Ebrahimi L, Hensley FW, Buechler MW, Debus J, Koch M, Weitz J, Bischof M: A clinical phase I/II trial to investigate preoperative dose-escalated intensity-modulated radiation therapy (IMRT) and intraoperative radiation therapy (IORT) in patients with retroperitoneal soft tissue sarcoma. BMC Cancer 2012, 12:287.

6. Dindo D, Demartines N, Clavien PA: Classification of surgical complications - a new proposal with evaluation in a cohort of 6336 patients and results of a survey. Ann Surg 2004, 240:205-213.

7. Jebsen NL, Trovik CS, Bauer HC, Rydholm A, Monge OR, Sundby Hall K, Alvegard T, Bruland OS: Radiotherapy to improve local control regardless of surgical margin and malignancy grade in extremity and trunk wall soft tissue sarcoma: a Scandinavian sarcoma group study. Int J Radiat Oncol Biol Phys 2008, 71:1196-1203.

8. Heslin MJ, Lewis JJ, Nadler E, Newman E, Woodruff JM, Casper ES, Leung D, Brennan MF: Prognostic factors associated with long-term survival for retroperitoneal sarcoma: implications for management. J Clin Oncol 1997, 15:2832-2839

9. Van de Voorde L, Delrue L, Van Eijkeren M, De Meerleer G: Radiotherapy and surgery - an indispensable duo in the treatment of retroperitoneal sarcoma. Cancer 2011, 117:4355-4364.

10. Gronchi A, Lo Vullo S, Fiore M, Mussi C, Stacchiotti S, Collini P, Lozza L, Pennacchioli E, Mariani L, Casali PG: Aggressive surgical policies in a retrospectively review single-institution case series of retroperitoneal soft tissue sarcoma patients. J Clin Oncol 2009, 27:24-30. 
11. Bonvalot S, Rivoire M, Castaing M, Stoeckle E, Le Cesne A, Blay JY, Laplanche A: Primary retroperitoneal sarcomas: a multivariate analysis of surgical factors associated with local control. J Clin Oncol 2009, 27:31-37.

12. Porter GA, Baxter NN, Pisters PW: Retroperitoneal sarcoma - a populationbased analysis of epidemiology, surgery, and radiotherapy. Cancer 2006, 106:1610-1616.

13. Stoeckle $E$, Coindre JM, Bonvalot $S$, Kantor G, Terrier P, Bonichon F, Nguyen Bui B, French Federation of Cancer Centers Sarcoma Group: Prognostic factors in retroperitoneal sarcoma: a multivariate analysis of a series of 165 patients of the French Cancer Center Federation Sarcoma Group. Cancer 2001, 92:359-368

14. Sampath S, Hitchcock YJ, Shrieve DC, Randall R, Schultheiss TE, Wong JY: Radiotherapy and extent of surgical resection in retroperitoneal softtissue sarcoma: multi-institutional analysis of 261 patients. J Surg Oncol 2010, 101:345-350.

15. Tepper JE, Suit HD, Wood WC, Proppe KH, Harmon D, McNulty P: Radiation therapy of retroperitoneal soft tissue sarcomas. Int J Radiat Oncol Biol Phys 1984, 10:825-830

16. Fein DA, Corn BW, Lanciano RM, Herbert SH, Hoffman JP, Coia LR: Management of retroperitoneal sarcomas: does dose escalation impact on locoregional control ? Int J Radiat Oncol Biol Phys 1995, 31:129-134.

17. Petersen IA, Haddock MG, Donohue JH, Nagorney DM, Grill J, Sargent DJ, Gunderson LL: Use of intraoperative electron beam radiotherapy in the management of retroperitoneal soft tissue sarcomas. Int J Radiat Oncol Biol Phys 2002, 52:469-475.

18. Gieschen HL, Spiro IJ, Suit HD, Ott MJ, Rattner DW, Ancukiewicz M, Wilett CG: Long-term results of intraoperative electron beam radiotherapy for primary and recurrent retroperitoneal soft tissue sarcoma. Int J Radiat Oncol Biol Phys 2001, 50:127-131.

19. Alektiar KM, Hu K, Anderson L, Brennan MF, Harrison LB: High-dose-rate intraoperative radiation therapy (HDR-IORT) for retroperitoneal sarcomas. Int J Radiat Oncol Biol Phys 2000, 47:157-163.

20. Shaw EG, Gunderson LL, Martin JK, Beart RW, Nagorney DM, Podratz KC: Peripheral nerve and ureteral tolerance to intraoperative radiation therapy: clinical and dose-response analysis. Radiother Oncol 1990, 18:247-255.

21. Paumier A, Le Pechoux C, Beaudre A, Negretti L, Ferreira I, Roberti E, Brahim J, Lefkopulos D, Daly-Schweitzer N, Bourhis J, Bonvalot S: IMRT or conformal radiotherapy for adjuvant treatment of retroperitoneal sarcoma? Radiother Oncol 2011, 99:73-78.

22. Llacer-Moscardo C, Quenet F, Azria D, Fenoglietto P: Feasibility study of volumetric modulated arc therapy for the treatment of retroperitoneal sarcomas. Radiat Oncol 2010, 5:83.

23. Sargos P, Dejean C, Figueiredo BH, Brouste V, Bui BN, Italiano A, Stoeckle E, Kantor G: High-dose pre-operative helical tomotherapy (54 Gy) for retroperitoneal liposarcoma. Radiat Oncol 2012, 7:214.

24. Gronchi A, De Paoli A, Dani C, Merlo DF, Quagliuolo V, Grignani G, Bertola G, Navarria P, Sangalli C, Buonadonna A, De Sanctis R, Sanfilippo R, Dei Tos AP, Stacchiotti S, Giorello L, Fiore M, Bruzzi P, Casali PG: Preoperative chemo-radiation therapy for localised retroperitoneal sarcoma: a phase I-II study from the Italian Sarcoma Group. Eur J Cancer 2014, 50:784-792.

25. Smith MJ, Ridgway PF, Catton CN, Cannell AJ, O'Sullivan B, Mikula LA, Jones $\mathrm{J}$, Swallow CJ: Combined management of retroperitoneal sarcoma with dose intensification radiotherapy and resection: long-term results of a prospective trial. Radiother Oncol 2014, 110:165-171.

26. Tzeng CW, Fiveash JB, Popple RA, Arnoletti JP, Russo SM, Urist MM, Bland Kl, Heslin MJ: Preoperative radiation therapy with selective dose escalation to the margin at risk for retroperitoneal sarcoma. Cancer 2006, 107:371-379.

27. McBride SM, Raut CP, Lapidus M, Devlin PM, Marcus KJ, Bertagnolli M, Geroge S, Baldini EH: Locoregional recurrence after preoperative radiation therapy for retroperitoneal sarcoma: adverse impact of multifocal disease and potential implications of dose escalation. Ann Surg Oncol 2013, 20:2140-2147.

28. Sweeting RS, Deal AM, Llaguna OH, Bednarski BK, Meyers MO, Yeh JJ, Calvo $\mathrm{BF}$, Tepper JE, Kim HJ: Intraoperative electron radiation therapy as an important treatment modality in retroperitoneal sarcoma. J Surg Res 2013, 185:245-249.

29. Alford S, Choong P, Chander S, Henderson M, Powell G, Ngan S: Outcomes of preoperative radiotherapy and resection of retroperitoneal sarcoma. ANZ J Surg 2013, 83:336-341.
30. Jones JJ, Catton CG, O'Sullivan B, Couture J, Heisler RL, Kandel RA, Swallow $\mathrm{CJ}$ : Initial results of a trial of preoperative external-beam radiation therapy and postoperative brachytherapy for retroperitoneal sarcoma. Ann Surg Oncol 2002, 9:346-354.

31. Pisters PW, Ballo MT, Fenstermacher MJ, Feig BW, Hunt KK, Raymond KA, Burgess MA, Zagars GK, Pollock RE, Benjamin RS, Patel SR: Phase I trial of preoperative cincurrent doxorubicin and radiation therapy, surgical resection and intraoperative electron-beam radiation therapy for patients with localized retroperitoneal sarcoma. J Clin Oncol 2003, 21:3092-3097.

32. Caudle AS, Tepper JE, Calvo BF, Meyers MO, Goyal LK, Cance WG, Kim HJ: Complications associated with neoadjuvant radiotherapy in the multidisciplinary treatment of retroperitoneal sarcomas. Ann Surg Oncol 2007, 14:577-582.

33. Zlotecki RA, Katz TS, Morris CG, Lind DS, Hochwald SN: Adjuvant radiation therapy for resectable retroperitoneal soft tissue sarcoma. Am J Clin Oncol 2005, 28:310-316.

34. Strauss DC, Hayes AJ, Thway K, Moskovic EC, Fisher C, Thomas JM: Surgical management of primary retroperitoneal sarcoma. Br J Surg 2010, 97:698-706.

35. Lewis JJ, Leung D, Woodruff JM, Brennan MF: Retroperitoneal soft-tissue sarcoma - analysis of 500 patients treated and followed at a single institution. Ann Surg 1998, 228:355-365.

36. Bonvalot S, Miceli R, Berselli M, Causeret S, Colombo C, Mariani L, Bouzaiene H, Le Pechoux C, Casali PG, Le Cesne A, Fiore M, Gronchi A: Aggressive surgery in retroperitoenal soft tissue sarcoma carried out at high-volume centers is safe and is associated with improved local control. Ann Surg Oncol 2010, 17:1507-1514.

37. Bartlett EK, Roses RE, Meise C, Fraker DL, Kelz RR, Karakousis GC: Preoperative Radiation for retroperitoneal sarcoma is not associated with increased early postoperative morbidity. J Surg Oncol 2014, 109:606-611.

38. Haddock MG, Miller RC, Nelson H, Pemberton JH, Dozois EJ, Alberts SR, Gundersson LL: Combined modality therapy including intraoperative electron irradiation for locally recurrent colorectal cancer. Int J Radiat Oncol Biol Phys 2011, 79:143-150.

\section{doi:10.1186/1471-2407-14-617}

Cite this article as: Roeder et al:: Clinical Phase I/II trial to Investigate Preoperative Dose-Escalated Intensity-Modulated Radiation Therapy (IMRT) and Intraoperative Radiation Therapy (IORT) in patients with retroperitoneal soft tissue sarcoma: interim analysis. BMC Cancer $201414: 617$

\section{Submit your next manuscript to BioMed Central and take full advantage of:}

- Convenient online submission

- Thorough peer review

- No space constraints or color figure charges

- Immediate publication on acceptance

- Inclusion in PubMed, CAS, Scopus and Google Scholar

- Research which is freely available for redistribution 